\title{
Deilmann, Clemens; Lehmann, Iris; Schumacher, Ulrich; Behnisch, Martin (Hrsg.) (2017): Stadt im Spannungsfeld von Kompaktheit, Effizienz und Umweltqualität. Anwendungen urbaner Metrik
}

\author{
Berlin/Heidelberg: Springer Spektrum. 231 Seiten, 87 Abbildungen, 44 Tabellen; \\ 7 Fallbeispiele mit zahlreichen Tabellen und Abbildungen; 17 Karten
}

\section{Otti Margraf ${ }^{1}$}

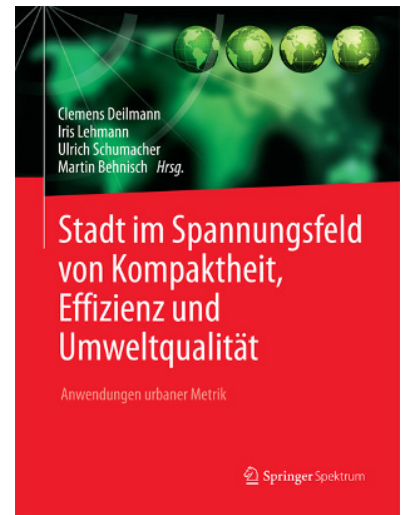

Mit dem vorliegenden Band zur Analyse und Bewertung stadträumlicher Strukturen wird der Versuch unternommen, Wirkungszusammenhänge der städtischen Physiognomie auf das räumliche Gefüge einer lebenswerten Stadt aufzuzeigen. Bei der Untersuchung von Ursache und Wirkung wird ein interessanter neuer methodischer Weg beschritten, indem primär von der vorgegebenen, inhaltlich charakterisierten, bewerteten und kartographierten Physiognomie und Struktur der Stadt ausgegangen wird, um ihre Auswirkung oder den Einfluss auf die Funktionalität der Stadt zu analysieren.

Diese Methodik basiert auf der so bezeichneten „urbanen Metrik“, bestehend aus einer zweidimensionalen Analysematrix (vgl. Kapitel 2). Während die Zeilen der Matrix zehn definierte Wirkungszusammenhänge (z. B. Erreichbarkeiten, Vielfalt des Wohnens, Zerschneidung des Siedlungskörpers, Erschließungseffizienz) beschreiben, werden die Spalten der Matrix als analytische Themenkom-

Prof. Dr. Otti Margraf

ottima48@gmail.com

1 Petunienweg 5, 04824 Beucha, Deutschland plexe über sinnstiftende Messgrößen und Indizes definiert (S. 16). Diese quantifizierbaren Größen charakterisieren Diversität, Formkomplexität, Heterogenität, Kernfläche, Nachbarschaft und Zerschneidung der räumlichen städtischen Struktur. Die Quantifizierung erfolgt grundsätzlich anhand des digitalen Basis-Landschaftsmodells (ATKISBasis-DLM) - vor allem mit den flächenhaft ausgewiesenen topographischen Objekten aus dem Objektbereich Siedlung. Außerdem werden bei mehreren Wirkungszusammenhängen die amtlichen Hausumringe aus dem Liegenschaftskataster sowie damit verknüpfte Bebauungsstrukturtypen verwendet, welche am Leibniz-Institut für ökologische Raumentwicklung (IÖR) erarbeitet wurden. Des Weiteren werden statistische Daten der Statistischen Landesämter, die INKAR-Indikatoren ${ }^{1}$ des Bundesinstituts für Bau-, Stadt- und Raumforschung (BBSR) oder der Stadtstatistischen Ämter vom KOSIS-Verbund ${ }^{2}$ miteinbezogen. Diese vorgestellten Zusammenhänge zwischen Form und Phänomen dienen auch der Weiterentwicklung des Monitorings von Raumeinheiten, also hier des Siedlungskörpers von Städten. Die Auswertung von Umweltqualitäts- sowie Kompaktheits- und Effizienzmerkmalen erfolgt mithilfe einer multikriteriellen Bewertung, deren Vergleichbarkeit durch die auf das Intervall $(0 ; 1)$ normierten Messgrößen zu einem Gesamtindex verknüpft und kartographisch visualisiert werden. Bei der multikriteriellen Bewertung der Städte anhand der normierten Messgrößen und deren additiver Verknüpfung werden die Unabhängigkeit bzw. fehlende korrelative Zusammenhänge zwischen den Messgrößen vorausgesetzt, ohne den klaren Nachweis beispielsweise anhand von Korrelationsanalysen zu erbringen. Auch die additive Verknüpfung sollte

${ }^{1}$ INKAR - Indikatoren und Karten zur Raum- und Stadtentwicklung.

${ }^{2}$ KOSIS - Kommunales Statistisches Informationssystem. 
man bei künftigen Beispielstudien auf ihre Sinnhaftigkeit überprüfen.

Die Auswahl der Städte Aachen, Dresden, Halle (Saale), Krefeld, Landau in der Pfalz, Neubrandenburg und Oldenburg erfolgte unter Berücksichtigung der Stadtgröße, der räumlichen Lage sowie der städtebaulichen Struktur und Entwicklung. Sie dienen als Fallbeispiele zur Umsetzung der angedachten Methodik. Sehr anschaulich und sehr gut graphisch aufbereitet erfolgt in Kapitel 3.2 die Charakterisierung der ausgewählten Städte anhand allgemeiner Kennzahlen (vergleichendes Netzdiagramm), Kennziffern zu Wirtschaft und Soziales (Bruttoinlandsprodukt (BIP), Altersstruktur, Bildung, Arbeitsplätze), zum Wohnen (Wohnungsbestand) und zur Flächen- und Bebauungsstruktur. Die sich am Kapitelende anschließende tabellarische Auflistung der verwendeten Kennzahlen (Definition, Berechnungsalgorithmus, Datenquelle und Zeitbezug) für die einzelnen Sachbereiche ermöglicht einen schnell erfassbaren Überblick über die gesamte Datenbasis.

Anhand dieser Datenbasis werden in Kapitel 4 die jeweils durch ein oder zwei Thesen untersetzten Wirkungszusammenhänge vorgestellt. Diese Thesen werden verbal formuliert und der quantifizierende Messansatz (z. B. flächengewichteter mittlerer Formindex) erläutert. Ausgewählte Beobachtungen werden mithilfe der Messwerte diskutiert und für die sieben ausgewählten Beispielstädte verglichen. Auch hier muss die nach einheitlichen Gesichtspunkten vorgenommene Gliederung, die tabellarische und bildhafte Ausgestaltung und Aufbereitung für alle Wirkungszusammenhänge hervorgehoben werden. Dies erleichtert ein schnelles Einlesen in die einzelnen Zusammenhangsvermutungen und den Vergleich der Ergebnisse. Zusätzlich liefert eine kartographisch sehr gut aufbereitete Kartenserie unter dem Titel „Physiognomie des Urbanen“ (S. 157) eine vergleichende kartographische Darstellung aller Städte zu den erläuterten und diskutierten 17 Thesen von Wirkungszusammenhängen. Sie runden das Bild von den beschriebenen und diskutierten Ergebnissen (karto-) graphisch sehr gut ab.
Nachdem sowohl die ausgewählten Städte im Detail als auch die Thesen der Wirkungszusammenhänge einzeln beschrieben, analysiert und diskutiert wurden, folgen abschließend noch eine Zusammenschau, Interpretationsbeispiele sowie eine kritische Diskussion der methodischen Bearbeitung und Analyse der ,urbanen Metrik“. Die Zusammenschau konzentriert sich dabei auf das im Buchtitel genannte Spannungsfeld der untersuchten städtischen Strukturen: Kompaktheit und Effizienz auf der einen Seite sowie Umweltqualität auf der anderen Seite. Sie liefert in tabellarisch und graphisch ebenfalls gut aufbereiteter und damit gut lesbarer Form einen systematischen Überblick der aggregierten und normierten Messwerte. Die Interpretationsbeispiele beschäftigen sich mit drei ausgewählten Themen: den potenziellen städtischen Flächen mit Hitzestress, der Ressourceneffizienz von Siedlungsstrukturen und der Wohnqualität.

In der vorliegenden Arbeit wird mit dem Begriff der urbanen Metrik ein Analyse- und Bewertungsansatz entwickelt und umgesetzt, der von der geometrischen Form und der räumlichen Struktur der Städte ausgeht. Mithilfe von klar definierten Messgrößen und Indizes werden Strukturen (Siedlungs-, Bebauungs-, Flächennutzungs- und Infrastrukturen) sowie Phänomene (Landschafts- und Ortsbilder, Bodenversiegelung, Emissionen und Immissionen sowie Ressourcen- und Energieverbrauch) untersucht. Die Analyse erfolgt anhand einer klaren, einheitlichen und damit gut vergleichbaren Vorgehensweise. Die textliche und graphische Aufbereitung der Untersuchungen anhand von Tabellen, Abbildungen, Fotos und Karten kann man nur immer wieder positiv hervorheben. Die Datenbereitstellung, Analyse und Darstellung der Ergebnisse erfolgt, wie bereits mehrfach angemerkt, nach klaren einheitlichen Kriterien, was der Lesbarkeit der Studie, insbesondere bei Vergleichen, sehr zugute kommt. Die klare Darstellung lässt auf eine leichte Umsetzung von Folgestudien und ,Nachahmern' hoffen, die andere Städtegruppen oder auch einen internationalen Städtevergleich ins Auge fassen. Alles in allem eine inhaltlich und methodisch sehr anregende Studie, die man mit steigendem Interesse liest. 